\title{
DILATION-COMMUTING OPERATORS ON POWER-WEIGHTED ORLICZ CLASSES
}

\author{
Ron Kerman, Rama Rawat and Rajesh K. Singh
}

Abstract. Let $\Phi$ be a nondecreasing function from $\mathbb{R}_{+}=(0, \infty)$ onto itself. Fix $\gamma \in \mathbb{R}=$ $(-\infty, \infty)$ and let $L_{\Phi, t} \gamma\left(\mathbb{R}_{+}\right)$be the set of all Lebesgue-measurable functions $f$ from $\mathbb{R}_{+}$to $\mathbb{R}$ for which

$$
\int_{\mathbb{R}_{+}} \Phi(k|f(t)|) t^{\gamma} d t<\infty
$$

for some $k>0$. Define the gauge $\rho_{\Phi, t \gamma}$ at $f \in L_{\Phi, t} \gamma\left(\mathbb{R}_{+}\right)$by

$$
\rho_{\Phi, t}(f)=\inf \left\{\lambda>0: \int_{\mathbb{R}_{+}} \Phi\left(\frac{|f(t)|}{\lambda}\right) \frac{t^{\gamma}}{\lambda} d t \leqslant 1\right\} .
$$

Our principal goal in this paper is to find conditions on the nondecreasing functions $\Phi_{1}$ and $\Phi_{2}, \gamma \in \mathbb{R}$ and an operator $T$ so that the assertions

$$
\rho_{\Phi_{1}, t} \gamma(T f) \leqslant C \rho_{\Phi_{2}, t} \gamma(f)
$$

and

$$
\int_{\mathbb{R}_{+}} \Phi_{1}(|(T f)(t)|) t^{\gamma} d t \leqslant K \int_{\mathbb{R}_{+}} \Phi_{2}(K|f(s)|) s^{\gamma} d s,
$$

concerning $f \in S\left(\mathbb{R}_{+}\right)$, the class of simple functions supported in $\mathbb{R}_{+}$, are equivalent and to then find necessary and sufficient conditions in order that $(\mathrm{M})$ holds.

In addition, we investigate the connection between $(\mathrm{G})$ and the assertion that

$$
T: \stackrel{\circ}{L}_{\Phi_{2}, t}\left(\mathbb{R}_{+}\right) \rightarrow L_{\Phi_{1}, t}\left(\mathbb{R}_{+}\right),
$$

where $\stackrel{\circ}{L}_{\Phi_{2}, t}\left(\mathbb{R}_{+}\right)$is the closure of $S\left(\mathbb{R}_{+}\right)$in $L_{\Phi_{2}, t}\left(\mathbb{R}_{+}\right)$.

Mathematics subject classification (2010): Primary 42B25, 26D15, Secondary 28A25.

Keywords and phrases: Dilation-commuting operators, Orlicz spaces, norm inequalities, modular inequalities, Hardy operator, maximal function, Hilbert transform.

\section{REFERENCES}

[1] S. Bloom And R. KeRman, Weighted $L_{\Phi}$ integral inequalities for operators of Hardy type, Studia Math. 110, 1 (1994), 35-52.

[2] S. Bloom And R. Kerman, Weighted Orlicz space integral inequalities for the Hardy-Littlewood maximal operator, Studia Math. 110, 2 (1994), 149-167.

[3] C. Bennett And R. Sharpley, Interpolation of operators, Pure and Applied Mathematics, vol. 129, Academic Press Inc., Boston, MA, 1988.

[4] A. Cianchi, Hardy inequalities in Orlicz spaces, Trans. Amer. Math. Soc. 351, 6 (1999), 2459-2478.

[5] R. COIFMAN AND C. FEFFERMAN, Weighted norm inequalities for maximal functions and singular integrals, Studia Math. 51, 3 (1974), 241-250.

[6] M. Fabian, P. Habala, P. HáJek, V. Montesinos and V. Zizler, Banach space theory, CMS Books in Mathematics/Ouvrages de Mathématiques de la SMC, Springer, 2011.

[7] R. KeRMAN AND L. PICK, Explicit formulas for optimal rearrangement-invariant norms in Sobolev imbedding inequalities, Studia Math. 206, 2 (2011), 97-119. 
[8] R. KERMAN AND A. TORCHINSKY, Integral inequalities with weights for the Hardy maximal function, Studia Math. 71, 3 (1982), 277-284.

[9] L. Maligranda, Orlicz spaces and interpolation, Sem. Math. 5, Dep. Mat., Univ. Estadual de Campinas, Campinas SP, Brazil (1989).

[10] W. Matuszewska And W. Orlicz, On certain properties of $\varphi$-functions, Bull. Acad. Polon. Sci. Sér. Sci. Math. Astronom. Phys. 8 (1960), 439-443.

[11] S. MAZUR AND W. ORLicz, On some classes of linear spaces, Studia Math. 17 (1958), 97-119.

[12] W. ORLicz, A note on modular spaces. I, Bull. Acad. Polon. Sci. Sér. Sci. Math. Astronom. Phys. 9 (1961), 157-162.

[13] A. TORChinsky, Real-variable methods in harmonic analysis, Pure and Applied Mathematics, vol. 123, Academic Press, Inc., Orlando, FL, 1986. 